\title{
Local recognition of the line graph of an anisotropic vector space
}

\author{
Kristina Altmann and Ralf Gramlich* \\ (Communicated by A. Pasini)
}

\begin{abstract}
Let $n \geq 7$ and let $V$ be an $(n+2)$-dimensional vector space endowed with an anisotropic sesquilinear form. In this article we characterise the graph whose vertices are the twodimensional subspaces of $V$ and in which two vertices are adjacent if and only if the corresponding two-dimensional spaces are perpendicular with respect to the sesquilinear form.
\end{abstract}

\section{Introduction}

A central problem in synthetic geometry is the characterisation of graphs and geometries. The local recognition of locally homogeneous graphs forms one category of such characterisations, which works as follows. Let $\Sigma$ be a graph. A graph $\Gamma$ is called locally $\Sigma$ if for each vertex $x$ of $\Gamma$ the graph $\Gamma_{x}$ is isomorphic to $\Sigma$, where $\Gamma_{x}$ is the induced subgraph of $\Gamma$ on the set of vertices adjacent to $x$. It is a natural question to ask for all isomorphism types of connected graphs which are locally some graph $\Sigma$. This classification question is called the local recognition problem for graphs that are locally $\Sigma$, which can be found in great quantities in the literature. One of the earliest and most influential is [3].

In the present article we focus on the line graph of an $n$-dimensional vector space over some field $\mathbb{F}$ endowed with an anisotropic sesquilinear form. Let $n \in \mathbb{N}$, let $V=V_{n}$ be an $n$-dimensional vector space over $\mathbb{F}$ and let $(\cdot, \cdot)$ be a scalar product on $V \times V$. For a subspace $U \subseteq V$ the polar of $U$ is $U^{\pi}=\{x \in V:(x, u)=0$ for all $u \in U\}$. The line graph $\mathbf{S}\left(V_{n}\right)$ of the vector space $V_{n}$ is the graph on the two-dimensional subspaces of $V_{n}$, the lines of $V_{n}$, where two distinct lines $l$ and $k$ of $V_{n}$ are adjacent, in symbols $k \perp l$, if and only if $l \subseteq k^{\pi}$ or, equivalently, if $k \subseteq l^{\pi}$. For a vertex $x$ of $\mathbf{S}\left(V_{n}\right)$ the local graph $\mathbf{S}\left(V_{n}\right)_{x}=: x^{\perp}$ is the subgraph induced by $\mathbf{S}\left(V_{n}\right)$ on the set of vertices $\left\{y \in \mathbf{S}\left(V_{n}\right)\right.$ $x \perp y\}$, the neighbours of $x$ in the graph $\mathbf{S}\left(V_{n}\right)$. For a set of vertices $X$ of $\mathbf{S}\left(V_{n}\right)$ the graph $X^{\perp}$ is defined as $\bigcap_{x \in X} x^{\perp}$.

\footnotetext{
${ }^{*}$ The second author gratefully acknowledges a Heisenberg fellowship by the Deutsche Forschungsgemeinschaft.
} 
The main result of this paper is the following local recognition theorem. Note that because of the size of the involved dimensions the field $\mathbb{F}$ is necessarily infinite.

Main Theorem. Let $n \geq 7$ and let $\Gamma$ be a connected locally $\mathbf{S}\left(V_{n}\right)$ graph. Then $\Gamma$ is isomorphic to $\mathbf{S}\left(V_{n+2}\right)$.

For $n=6$ there exist counterexamples to the statement of the Main Theorem arising from anisotropic twisted forms of the semisimple algebraic group $E_{6}(\mathbb{F})$. In the first author's $\mathrm{PhD}$ thesis [1] the situation $n=6$ is dealt with for the field $\mathbb{F}=\mathbb{C}$. Theorem 4.1.2 of [1] is an analogue of our main theorem for that case under a mild extra assumption. We would like to stress that the case $n=6$ is much more difficult than the case $n \geq 7$ presented here. The proof given in [1] is based on a construction of a large automorphism group of the graph $\Gamma$, the existence of a subgraph of $\Gamma$ isomorphic to the reflection graph of the Coxeter groups of type $A_{7}$, respectively $E_{6}$ (this makes serious use of [3]), and a recognition of the automorphism group via Lie theory and Phan theory (cf. [7]).

In Section 2 we discuss group-theoretic applications of our main result. Sections 3 and 4 provide some basic facts about the reconstruction of $\mathbb{P}\left(V_{n}\right)$ from $\mathbf{S}\left(V_{n}\right)$ and how the induced subgraphs $x^{\perp}, x \in \mathbf{S}\left(V_{n}\right)$, relate to codimension two subspaces of $\mathbb{P}\left(V_{n}\right)$. Finally, in Section 5 we prove the main result. The strategy used in that section is the same as in [4], [6].

\section{Group-theoretic consequences}

Assume that the automorphism group of $\mathbf{S}\left(V_{n}\right)$ acts transitively on the set of ordered triangles of $\mathbf{S}\left(V_{n}\right)$, as is the case for $\mathbb{F}=\mathbb{C}$ and the standard scalar product. (We refer the reader to [5] for a detailed investigation of fields admitting such a transitivity.) Then our main result implies the following group-theoretic recognition theorem.

Local Recognition Theorem. Let $n \geq 7$, let $\mathbb{F}$ be a field admitting an anisotropic sesquilinear form such that the automorphism group of $\mathbf{S}\left(V_{n}\right)$ acts transitively on the set of ordered triangles of $\mathbf{S}\left(V_{n}\right)$, and let $G$ be a group containing an involution $x$ and a subgroup $K \unlhd C_{G}(x)$ such that

1. $K \cong \mathrm{SU}_{n}(\mathbb{F})$;

2. $C_{G}(K)$ contains a subgroup $X \cong \mathrm{SU}_{2}(\mathbb{F})$ with $x=Z(X)$;

3. there exists an involution $g \in G$ such that $Y:=g X g$ is contained in $K$;

4. if $V$ is a natural module for $K$, then the commutator $[Y, V]=$ $\{y v-v \in V \mid y \in Y, v \in V\}$ has $\mathbb{F}$-dimension two;

5. $G=\langle K, g K g\rangle$; moreover, there exists $z \in K \cap g K g$ which is a $g K g$-conjugate of $x$ and a $K$-conjugate of $g x g$.

Then $G / Z(G) \cong \operatorname{PSU}_{n+2}(\mathbb{F})$.

Proof. Define $y:=g x g$ and $J:=g K g$. By 4. the group $X$ is a fundamental subgroup of $J$ with centre $x$ and $Y$ is a fundamental subgroup of $K$ with centre $y$. Since the group $K$ is isomorphic to $\mathrm{SU}_{n}(\mathbb{F})$ and since $y$ and $z$ are centres of fundamental subgroups of $K$ with 
$[y, z]=1$, we find the elements $y$ and $z$ to be conjugate in $K$ by an involution. Similarly $x$ and $z$ are conjugate in $J$ by an involution. Therefore the normaliser of $\{x, y, z\}$ with respect to the conjugation action of the group $G$ equals the group $\mathrm{Sym}_{3}$. Hence the normaliser of $\{x, y\}$ is the group $\mathrm{Sym}_{2}$.

Consider the graph $\Gamma$ on all conjugates of $x$ in $G$ in which two vertices $a, b$ are adjacent if there exists an element $h \in G$ such that $\left(h x h^{-1}, h y h^{-1}\right)=(a, b)$. Since $G$ induces the action of $\mathrm{Sym}_{3}$ on $\{x, y, z\}$, this definition of adjacency is completely symmetric, and we have defined an undirected graph $\Gamma$ in which the elements $x, y, z$ form a triangle. The stabiliser of $\{x, y\}$ permutes $x$ and $y$ and therefore interchanges $C_{G}(x) \unrhd K$ and $C_{G}(y) \unrhd J$. Hence the stabiliser of $x$ together with the stabiliser of $\{x, y\}$ generates $G$, as $G=\langle J, K\rangle \leq\left\langle C_{G}(x), C_{G}(y)\right\rangle$. Consequently, the graph $\Gamma$ is connected.

Moreover, $\Gamma$ is a locally isomorphic to a line graph by construction. To prove this, it is enough to show that any triangle in $\Gamma$ is a conjugate of $(x, y, z)$. Let $(a, b, c)$ be a triangle and let $h \in G$ with $\left(h x h^{-1}, h y h^{-1}\right)=(a, b)$. Notice that the elements $b=h y h^{-1}$, $d:=h z h^{-1}$ of $h K h^{-1}$ commute. The edges $(a, b)$ and $(a, c)$ are both conjugate in $G$ to $(x, y)$, so that they are conjugate to each other by an element of $C_{G}(a) \unrhd h K h^{-1}$. Since

$b \in h K h^{-1}$, we have $c \in h K h^{-1}$ as well. We have proved that $b, c, d$ are centres of fundamental subgroups of $h K h^{-1}$. Since $[b, c]=1=[b, d]$, the elements $c$ and $d$ are conjugate by an element of $C_{h K h^{-1}}(b)$. Therefore $(a, b, c)$ and $(x, y, z)$ are conjugate in $G$. Hence by the Main Theorem $\Gamma$ is isomorphic to $\mathbf{S}\left(V_{n+2}\right)$ and the claim follows.

There is an analogue of the above theorem for finite fields. See [2] for the case $n \geq 7$ and [8, Section 7.2.1] for the case $n=6$.

\section{Reconstruction of projective space}

In this section we collect some basic properties of the line graphs of the unitary vector spaces $V_{n}$ for $n \geq 5$, such as their diameters, cf. Proposition 3.2 and Proposition 3.3. We will also construct from $\mathbf{S}\left(V_{n}\right)$ a point-line geometry $\mathcal{G}=(\mathcal{P}, \mathcal{L}, \supset)$ which is isomorphic to the projective space given by the points and lines of $V_{n}$.

Since the behaviours of $V_{5}$ and $V_{n}, n \geq 6$, are slightly different, we will consider these graphs separately where appropriate.

Lemma 3.1. Let $l$ and $m$ be distinct lines of $V_{5}$. Then the distance of $l$ and $m$ in $\mathbf{S}\left(V_{5}\right)$ is

- one if and only if $l \subseteq m^{\pi}$ or $m \subseteq l^{\pi}$;

- two if and only if $\langle l, m\rangle$ is a three-dimensional subspace of $V_{5}$;

- three if and only if $l$ and $m$ do not intersect, are not perpendicular and satisfy that the intersection $l^{\pi} \cap m$ is one-dimensional;

- four if and only if $l$ and $m$ do not intersect and satisfy that the intersection $l^{\pi} \cap m$ is trivial.

Proof. These claims are straightforward to prove. We leave this as an exercise to the reader. 
Proposition 3.2. The graph $\mathbf{S}\left(V_{5}\right)$ is connected of diameter four. Moreover, the graph $\mathbf{S}\left(V_{5}\right)$ is locally $\mathbf{S}\left(V_{3}\right)$.

Proof. This is immediate from Lemma 3.1, because any pair of distinct lines $l$ and $m$ belongs to one of the four cases considered in 3.1 .

Proposition 3.3. Let $n \geq 6$. Any two elements of $\mathbf{S}\left(V_{n}\right)$ have a common neighbour. In particular, the graph $\mathbf{S}\left(V_{n}\right)$ is connected of diameter two.

Proof. Let $l$ and $m$ be two different two-dimensional subspaces of $V_{n}, n \geq 6$. The dimension formula implies $\operatorname{dim}\left(l^{\pi} \cap m^{\pi}\right)=\operatorname{dim}\left(l^{\pi}\right)+\operatorname{dim}\left(m^{\pi}\right)-\operatorname{dim}\left(\left\langle l^{\pi}, m^{\pi}\right\rangle\right) \geq$ $n-4 \geq 2$. Hence, in $V_{n}$ there exists a two-dimensional subspace $h \subseteq l^{\pi} \cap m^{\pi}$.

Proposition 3.4. For $n \geq 6$, the graph $\mathbf{S}\left(V_{n}\right)$ is locally $\mathbf{S}\left(V_{n-2}\right)$.

Proof. For each vertex $l$ of $\mathbf{S}\left(V_{n}\right)$, the space $l^{\pi} \subseteq V_{n}$ is an $(n-2)$-dimensional vector space endowed with an anisotropic sesquilinear form $\left.(\cdot, \cdot)\right|_{\mid l^{\pi} \times l^{\pi}}$. The claim follows.

Definition 3.5. Let $U$ be a subspace of the unitary vector space $V_{n}$. The set of all lines in $U$ is denoted by $\mathbf{L}(U)$.

Lemma 3.6. Let $l$ and $m$ be vertices of $\mathbf{S}\left(V_{n}\right)$ with $\{l, m\}^{\perp} \neq \emptyset$. Then the vertex set of the graph $\{l, m\}^{\perp \perp}$ equals the line set $\mathbf{L}(\langle l, m\rangle)$.

Proof. Since the vertex set of $z^{\perp}$ equals $\mathbf{L}\left(z^{\pi}\right)$, we have $\{l, m\}^{\perp \perp}=\left(\{l, m\}^{\perp}\right)^{\perp}=$ $\bigcap_{z \in\{l, m\}^{\perp}} z^{\perp}=\bigcap_{z \in\{l, m\}^{\perp}} \mathbf{L}\left(z^{\pi}\right)$. Moreover, for every $z \in\{l, m\}^{\perp}$ we have $\mathbf{L}(\langle l, m\rangle)$ $\subseteq \mathbf{L}\left(z^{\pi}\right)$. Therefore the set $\mathbf{L}(\langle l, m\rangle)$ is a subset of the vertex set of $\{l, m\}^{\perp \perp}$.

Conversely, let $p$ be a one-dimensional subspace not contained in $\langle l, m\rangle$. This implies $\langle l, m\rangle^{\pi} \nsubseteq p^{\pi}$ and $\langle l, m\rangle \subseteq p^{\pi}$. Let $t$ be a one-dimensional subspace of $\langle l, m\rangle^{\pi}$ which is not contained in $p^{\pi}$.

By assumption $3 \leq \operatorname{dim}(\langle l, m\rangle) \leq 4$ if $n \geq 6$ and $\operatorname{dim}(\langle l, m\rangle)=3$ if $n=5$, thus we have $\operatorname{dim}\left(\langle l, m\rangle^{\pi} \cap p^{\pi}\right)=\operatorname{dim}\left(\langle l, m\rangle^{\pi}\right)+\operatorname{dim}\left(p^{\pi}\right)-\operatorname{dim}\left(\left\langle p^{\pi},\langle l, m\rangle^{\pi}\right\rangle\right)=$ $\operatorname{dim}\left(\langle l, m\rangle^{\pi}\right)-1 \geq 1$. Hence there exists a one-dimensional subspace $s \subseteq\langle l, m\rangle^{\pi} \cap p^{\pi}$. The two-dimensional subspace $h=\langle s, t\rangle$ is a vertex of the graph $\{l, m\}^{\perp}$, so $\{l, m\}^{\perp \perp} \subseteq$ $h^{\perp}=\mathbf{L}\left(h^{\pi}\right) \subseteq \mathbf{L}\left(p^{\pi}\right)$. Thus $\{l, m\}^{\perp \perp}$ cannot contain two-dimensional subspaces containing $p$ as no line of $\mathbf{L}\left(p^{\pi}\right)$ contains the point $p$.

The claim follows because $p$ is an arbitrary one-dimensional subspace not contained in $\langle l, m\rangle$.

A similar statement can be proved about three different vertices under some additional assumptions.

Lemma 3.7. Let $k, l$ and $m$ be three distinct vertices of $\mathbf{S}\left(V_{n}\right)$. Suppose $k \cap l \cap m$ contains a one-dimensional subspace of $V_{n}$ and suppose that $\{k, l, m\}^{\perp} \neq \emptyset$. Then $\mathbf{L}(\langle k, l, m\rangle)=\{k, l, m\}^{\perp \perp}$. 
Proof. Since the lines $k, l$ and $m$ intersect in a common point in $V_{n}$, the dimension of $\langle k, l, m\rangle$ equals three or four.

If $\langle k, l, m\rangle$ is a three-dimensional subspace, then $m$ is contained in $\langle k, l\rangle$ and $\langle k, l, m\rangle$ $=\langle k, l\rangle$. From Lemma 3.6 it follows that $\mathbf{L}(\langle k, l, m\rangle)=\mathbf{L}(\langle k, l\rangle)=\{k, l\}^{\perp \perp}=$ $\{k, l, m\}^{\perp \perp}$ as $\emptyset \neq\{k, l, m\}^{\perp} \subseteq\{k, l\}^{\perp}$.

In case $\operatorname{dim}(\langle k, l, m\rangle)=4$ pick a line $s$ in $\langle k, l, m\rangle$. For $n \geq 6$ we consider the two-dimensional orthogonal subspace $t=s^{\pi} \cap\langle k, l, m\rangle$. By Lemma 3.6 again we have $\mathbf{L}(\langle k, l, m\rangle)=\mathbf{L}(\langle s, t\rangle)=\{s, t\}^{\perp \perp}$. Hence the claim follows by

$$
\begin{aligned}
\{k, l, m\}^{\perp \perp}=\left(\{k, l, m\}^{\perp}\right)^{\perp} & =\bigcap_{z \in\{k, l, m\}^{\perp}} z^{\perp}=\bigcap_{z \in \mathbf{L}\left(\langle k, l, m\rangle^{\pi}\right)} z^{\perp}=\bigcap_{z \in \mathbf{L}\left(\langle s, t\rangle^{\pi}\right)} z^{\perp} \\
& =\bigcap_{z \in\{s, t\}^{\perp}} z^{\perp}=\left(\{s, t\}^{\perp}\right)^{\perp}=\{s, t\}^{\perp \perp} .
\end{aligned}
$$

If $n=5$ and $\operatorname{dim}(\langle k, l, m\rangle)=4$, then the orthogonal subspace $\langle k, l, m\rangle^{\pi}$ is of dimension one. This implies that $\{k, l, m\}^{\perp}$ is the empty graph, so we are done.

Remark 3.8. Suppose $l$ and $m$ are distinct lines which intersect in a common point in $V_{5}$. Then $\operatorname{dim}(\langle l, m\rangle)=3$, which implies that $\{l, m\}^{\perp} \neq \emptyset$ using Lemma 3.1. Moreover by Lemma 3.6 the vertex set of the induced subgraph $\{l, m\}^{\perp \perp}$ in $\mathbf{S}\left(V_{5}\right)$ equals the line set $\mathbf{L}(\langle l, m\rangle)$ and therefore the double perp $\{l, m\}^{\perp \perp}$ is minimal with respect to inclusion (i.e. for any distinct vertices $s_{1}, s_{2}$ in the double perp $\{l, m\}^{\perp \perp}$ we have the equality $\left.\left\{s_{1}, s_{2}\right\}^{\perp \perp}=\{l, m\}^{\perp \perp}\right)$.

Lemma 3.9. Two different lines $l$ and $m$ of $V_{n}$, for $n \geq 6$, intersect in a common point if and only if the double perp $\{l, m\}^{\perp \perp}$ in $\mathbf{S}\left(V_{n}\right)$ is minimal with respect to inclusion (i.e. for any distinct vertices $s_{1}, s_{2} \in\{l, m\}^{\perp \perp}$ we have $\left.\left\{s_{1}, s_{2}\right\}^{\perp \perp}=\{l, m\}^{\perp \perp}\right)$.

Proof. Certainly, if $l$ and $m$ are two different intersecting lines in $V_{n}$, then $\operatorname{dim}(\langle l, m\rangle)=$ 3. For any two distinct elements $s_{1}, s_{2} \in\{l, m\}^{\perp \perp}=\mathbf{L}(\langle l, m\rangle)$, the subspace $\left\langle s_{1}, s_{2}\right\rangle$ has dimension three implying that $\left\langle s_{1}, s_{2}\right\rangle=\langle l, m\rangle$. By Lemma 3.6, $\mathbf{L}(\langle l, m\rangle)=\{l, m\}^{\perp \perp}$ and $\mathbf{L}\left(\left\langle s_{1}, s_{2}\right\rangle\right)=\left\{s_{1}, s_{2}\right\}^{\perp \perp}$, thus $\left\{s_{1}, s_{2}\right\}^{\perp \perp}=\mathbf{L}\left(\left\langle s_{1}, s_{2}\right\rangle\right)=\mathbf{L}(\langle l, m\rangle)=\{l, m\}^{\perp \perp}$.

Suppose $\operatorname{dim}(\langle l, m\rangle)=4$ then $l$ and $m$ are two non-intersecting lines in $V_{n}$. Let $h \in \mathbf{L}(\langle l, m\rangle)$ such that $\operatorname{dim}(h \cap l)=1$. Then the span of $h$ and $l$ is of dimension three and $\{h, l\}^{\perp \perp}=\mathbf{L}(\langle h, l\rangle) \varsubsetneqq \mathbf{L}(\langle l, m\rangle)=\{l, m\}^{\perp \perp}$ by Lemma 3.6, which shows that the double perp $\langle l, m\rangle^{\perp \perp}$ is not minimal with respect to inclusion.

As mentioned in the beginning of this section, our strategy is to reconstruct the projective space on the one-dimensional and two-dimensional subspaces of $V_{n}$ from the graph $\mathbf{S}\left(V_{n}\right)$. The two-dimensional subspaces are easily recovered; we can simply take the vertex set of $\mathbf{S}\left(V_{n}\right)$ as the line set of the geometry. In a projective space of sufficient dimension each point is uniquely determined by the set of lines incident with it. We call such a set a line pencil. A successful description of line pencils essentially depends on the understanding when three lines intersect in a common point. Again we have to distinguish the cases $n=5$ and $n \geq 6$. 
Three different pairwise intersecting lines $k_{1}, k_{2}, k_{3}$ of the vector space $V_{5}$ intersect in one point if we can find a line $s$ in $V_{5}$ such that

- the line $s$ intersects the line $k_{i}$, if $s \neq k_{i}$, for $1 \leq i \leq 3$,

- $\left\langle s, k_{1}, k_{2}\right\rangle$ is a four-dimensional subspace in $V_{5}$.

This observation motivates the following definition in terms of double perps.

Definition 3.10. Let $\Gamma$ be a graph isomorphic to $\mathbf{S}\left(V_{5}\right)$. Two different vertices $k$ and $l$ of $\Gamma$ are defined to intersect if the induced subgraph $\{k, l\}^{\perp}$ is not empty. Three different pairwise intersecting vertices $k_{1}, k_{2}$ and $k_{3}$ of $\Gamma$ are defined to intersect in one point if the graph $\Gamma$ contains a vertex $s$ with the following properties:

- the vertex $s$ intersects each vertex $k_{i}$, if $s \neq k_{i}$, for $1 \leq i \leq 3$,

- the graph $\left\{k_{1}, k_{2}, s\right\}^{\perp}$ is empty.

An interior point of the graph $\Gamma$ is a maximal set $\mathcal{S}$ of different pairwise intersecting vertices of $\Gamma$ such that any three elements of $\mathcal{S}$ intersect in one point. These maximal sets exist by Zorn's lemma. Moreover, it is easily seen that every point of $V_{5}$ can in fact be realised by three pairwise intersecting lines. We denote the set of all interior points of $\Gamma$ by $\mathcal{P}$. Moreover, an interior line of the graph $\Gamma$ is a vertex of the graph $\Gamma$. The set of all interior lines of $\Gamma$ is denoted by $\mathcal{L}$.

For $n \geq 6$ the geometric properties of three different pairwise intersecting lines have a slightly different meaning in terms of double perps.

Definition 3.11. Let $n \geq 6$ and $\Gamma$ be graph isomorphic to the line graph $\mathbf{S}\left(V_{n}\right)$. Two different vertices $k$ and $l$ of $\Gamma$ are defined to intersect if the double perp $\{k, l\}^{\perp \perp}$ is minimal in $\Gamma$ with respect to inclusion (among double perps of distinct vertices). Three distinct pairwise intersecting vertices $k_{1}, k_{2}$ and $k_{3}$ of $\Gamma$ are defined to intersect in one point if there is a vertex $s$ in $\Gamma$ satisfying the following conditions:

- the vertex $s$ intersects $k_{i}$, if $s \neq k_{i}$, for $1 \leq i \leq 3$,

- the graph $\left\{k_{1}, k_{2}, s\right\}^{\perp}$ is non-empty and $\left\{k_{1}, k_{2}\right\}^{\perp \perp}=\mathbf{L}\left(\left\langle k_{1}, k_{2}\right\rangle\right) \varsubsetneqq \mathbf{L}\left(\left\langle k_{1}, k_{1}, s\right\rangle\right)$ $=\left\{k_{1}, k_{2}, s\right\}^{\perp \perp}$.

An interior point of the graph $\Gamma$ is a maximal set $\mathcal{S}$ of distinct pairwise intersecting vertices of $\Gamma$ such that any three elements of $\mathcal{S}$ intersect in one point. We denote the set of all interior points of $\Gamma$ by $\mathcal{P}$. Moreover, an interior line of the graph $\Gamma$ is a vertex of the graph $\Gamma$. The set of all interior lines of $\Gamma$ is denoted by $\mathcal{L}$.

By construction we obtain the following:

Proposition 3.12. Let $n \geq 5$ and let $\Gamma$ be a graph isomorphic to $\mathbf{S}\left(V_{n}\right)$. The geometry $\mathcal{G}=(\mathcal{P}, \mathcal{L}, \supset)$ on the interior points and interior lines of $\Gamma$ is isomorphic to the projective space of the vector space $V_{n}$.

For a graph $\Gamma \cong \mathbf{S}\left(V_{n}\right)$ we call the space $\mathcal{G}=(\mathcal{P}, \mathcal{L})$ the interior space on $\Gamma$. Note that this interior space carries a canonical polarity $\pi$ given by $l \mapsto l^{\perp}$. Hence we also obtain information about the automorphism group of $\mathbf{S}\left(V_{n}\right)$. 
Corollary 3.13. Let $n \geq 5$. The automorphism group of $\mathbf{S}\left(V_{n}\right)$ is isomorphic to the automorphism group of the projective space of $\left(V_{n},(\cdot, \cdot)\right)$.

\section{Subspaces arising from local graphs}

Let $n \geq 7$ and let $\Gamma \cong \mathbf{S}\left(V_{n}\right)$. Proposition 3.12 allows us to reconstruct a projective space $\mathcal{G}=(\mathcal{P}, \mathcal{L})$ isomorphic to $\mathbb{P}\left(V_{n}\right)$ from $\Gamma$. By Proposition 3.4 the graph $\Gamma$ is locally $\mathbf{S}\left(V_{n-2}\right)$. Hence for a vertex $x$ of $\Gamma$ Proposition 3.12 also allows us to reconstruct a projective space $\mathcal{G}_{x}=\left(\mathcal{P}_{x}, \mathcal{L}_{x}\right)$ isomorphic to $\mathbb{P}\left(V_{n-2}\right)$ from the induced subgraph $x^{\perp}$. The purpose of this section is to show that there is a canonical way to consider interior space $\mathcal{G}_{x}$ as a subspace of $\mathcal{G}$. We will spell out the details for the more complicated case $n=7$ and leave the easier case $n \geq 8$ as an exercise to the reader.

Notation. Every object of the interior space $\mathcal{G}_{x}$ will be indexed by the vertex $x$. In particular, for vertices $l, k, m$ of the subgraph $x^{\perp}$ we use the notation $\{k, l, m\}_{x}^{\perp}:=$ $\{k, m, l\}^{\perp} \cap x^{\perp}$ and $\{k, l, m\}_{x}^{\perp \perp}:=\left(\{k, l, m\}_{x}^{\perp}\right)_{x}^{\perp}=\left(\{k, l, m\}_{x}^{\perp}\right)^{\perp} \cap x^{\perp}$.

Obviously the line set $\mathcal{L}_{x}$ is properly contained in the line set $\mathcal{L}$. Therefore again we only have to understand the behaviour of the interior points.

Lemma 4.1. Let $p$ be a point of $\mathcal{G}$ and let $l, m \in p \cap \mathcal{L}_{x}$. Then the lines $l$ and $m$ intersect each other in $\mathcal{G}_{x}$.

Proof. The claim follows from the second item of Lemma 3.1 once we have shown that $\{l, m\}_{x}^{\perp} \neq \emptyset$.

Since $l, m \in x^{\perp}$ and $\{l, m\}^{\perp \perp}$ is minimal with respect to inclusion, the threedimensional subspace $\langle l, m\rangle$ is contained in $x^{\pi}$. Thus $\operatorname{dim}\left(x^{\pi} \cap\langle l, m\rangle^{\pi}\right)=2$ implying that $\{l, m\}_{x}^{\perp}=\{l, m, x\}^{\perp} \neq \emptyset$.

Lemma 4.2. Let $p$ be a point in $\mathcal{G}$ and let $k_{1}, k_{2}, k_{3} \in p \cap \mathcal{L}_{x}$ be pairwise distinct elements. Then the corresponding interior lines intersect in a common point of $\mathcal{G}_{x}$.

Proof. By Definition 3.10 and Lemma 4.1 it suffices to find a vertex $s \in x^{\perp}$ such that

- for each $i \in\{1,2,3\}$, the graph $\left\{s, k_{i}\right\}_{x}^{\perp}$ is not empty,

- the graph $\left\{s, k_{1}, k_{2}\right\}_{x}^{\perp}$ is empty.

The vertices $k_{1}, k_{2}$ and $k_{3}$ are adjacent to the vertex $x$, thus $\left\langle k_{1}, k_{2}, k_{3}\right\rangle \subseteq x^{\pi}$ in $\mathcal{G}$.

If $\left\langle k_{1}, k_{2}, k_{3}\right\rangle$ is a four-dimensional subspace of $\mathcal{G}$, then one can choose $s=k_{3}$. Then $\left\langle k_{1}, k_{2}, s\right\rangle^{\pi} \cap x^{\pi}$ is a point $z$ and any line $l$ of $\left\langle k_{1}, k_{2}, s\right\rangle^{\pi}$ intersects $x^{\pi}$ in the point $z$ or not at all. Thus $\left\{k_{1}, k_{2}, s\right\}_{x}^{\perp}=\emptyset$.

Hence suppose $\left\langle k_{1}, k_{2}, k_{3}\right\rangle$ is three-dimensional. By assumption $k_{1}, k_{2}$ and $k_{3}$ are elements of the point $p \in \mathcal{P}$. Since each of the $k_{i}$ is a neighbour of $x$, the point $k_{1} \cap$ $k_{2} \cap k_{3}=d$ in fact lies in $x^{\pi}$. Fix a point $y$ in $\left\langle k_{1}, k_{2}, k_{3}\right\rangle^{\pi} \cap x^{\pi}$ and consider the line $s=\langle d, y\rangle$. The line $s$ is contained in $x^{\pi}$ and intersects each line $k_{i}, i \in\{1,2,3\}$, in the point $d$. By Lemma 3.1, the graph $\left\{s, k_{i}\right\}_{x}^{\perp}$ is not empty. Since $y \nsubseteq\left\langle k_{1}, k_{2}\right\rangle$, the 
subspace $\left\langle s, k_{1}, k_{2}\right\rangle$ has dimension four, and it follows, using the arguments from above, that $\left\{s, k_{1}, k_{2}\right\}_{x}^{\perp}$ is empty.

Proposition 4.3. Let $p$ be a point in $\mathcal{G}$ with $p \cap \mathcal{L}_{x} \neq \emptyset$. Then $p \cap \mathcal{L}_{x}$ is a point of $\mathcal{G}_{x}$.

Proof. By Lemma 4.1 and Lemma 4.2, the intersection $p \cap \mathcal{L}_{x}$ is a subset of some interior point $p_{x}$ if $\left|p \cap \mathcal{L}_{x}\right| \geq 2$.

Let $l \in p \cap \mathcal{L}_{x}$, then $l \cap m \subseteq x^{\pi}$ for each element $m \in p$. As $\operatorname{dim}\left(x^{\pi}\right)=5$ we can find a two-dimensional subspace $n$ properly contained in $x^{\pi}$ such that $l \cap n=l \cap m$. Certainly $n$ is an element of $p$ implying that $\left|p \cap \mathcal{L}_{x}\right| \geq 2$.

To complete the proof of the statement, it remains to show that each $k \in p_{x}$ satisfies $k \in p$. The vertex $k$ corresponds to a line in the subspace $x^{\pi}$ of $\mathcal{G}$ incident to the point $d=l \cap m$ for two different elements $l, m \in p$.

Furthermore, for each $n \in p$ different from $k$, the space spanned by $k$ and $n$ is three-dimensional as $n$ intersects $k$ in the point $d$. Lemma 3.9 implies that $\{k, n\}^{\perp \perp}$ is minimal with respect to inclusion, which is the first condition of Definition 3.11. To establish the second condition of this definition, let $n, t \in p$ be distinct lines and different from $k$. The lines $k, n, t$ mutually intersect, by the arguments from above, so the subspace $\langle k, n, t\rangle$ has dimension three or four. If $\operatorname{dim}(\langle k, n, t\rangle)=4$, then $\{k, n\}^{\perp \perp}=$ $\mathbf{L}(\langle k, n\rangle) \varsubsetneqq \mathbf{L}(\langle k, n, t\rangle)=\{k, n, t\}^{\perp \perp}$ by Lemma 3.6 and Lemma 3.7. If $\langle k, n, t\rangle$ is three-dimensional, then $\langle k, n, t\rangle=\langle k, n\rangle$. Therefore there certainly exists a line $s$ intersecting the lines $k, n, t$ in the point $d$ such that $\langle k, n, s\rangle$ is a four-dimensional space in $\mathcal{G}$ and $\{k, n\}^{\perp \perp}=\mathbf{L}(\langle k, n\rangle) \varsubsetneqq \mathbf{L}(\langle k, n, s\rangle)=\{k, n, s\}^{\perp \perp}$ by Lemma 3.6 and Lemma 3.7. Hence $k \in p$, which proves the claim.

Next we show that each point $p_{x}$ of the interior space $\mathcal{G}_{x}$ on $x^{\perp}$ is contained in a unique point $p$ of the interior space $\mathcal{G}$ of $\Gamma$.

Lemma 4.4. Let $p_{x}$ be a point in $\mathcal{G}_{x}$ and let $k, l$ be two distinct elements of $p_{x}$. Then $k$ and $l$ intersect in $\mathcal{G}$.

Proof. By Definition 3.11 we have to check that $\{k, l\}^{\perp \perp}$ is minimal with respect to inclusion. By Lemma 3.9 this is the case if and only if the span of $k$ and $l$ is threedimensional subspace. By way of contradiction assume that $k$ and $l$ span a four-dimensional subspace. Since $k, l \in p_{x}$, thus $\langle k, l\rangle \cap x$ has dimension zero and therefore the subspace $\langle k, l, x\rangle$ is of dimension six. Hence $\operatorname{dim}\left(\langle k, l, x\rangle^{\pi}\right)=1$, which means that $\{k, l, x\}^{\perp}=\{k, l\}^{\perp} \cap x^{\perp}=\emptyset$, a contradiction to $k, l \in p_{x}$, cf. Definition 3.10.

Lemma 4.5. Let $p_{x}$ be a point in $\mathcal{G}_{x}$ and let $k_{1}, k_{2}, k_{3}$ three different interior lines of $p_{x}$. Then the corresponding interior lines of $\mathcal{G}$ intersect in a common point.

Proof. By Lemma 4.4, it remains to show, cf. Definition 3.11, that there is a vertex $s$ of $\Gamma \cong \mathbf{S}\left(V_{7}\right)$ such that

- for $i \in\{1,2,3\}$, if $s \neq k_{i}$, the double perp $\left\{s, k_{i}\right\}^{\perp \perp}$ is minimal with respect to inclusion,

- $\left\{k_{1}, k_{2}\right\}^{\perp \perp}=\mathbf{L}\left(\left\langle k_{1}, k_{2}\right\rangle\right) \varsubsetneqq \mathbf{L}\left(\left\langle k_{1}, k_{2}, s\right\rangle\right)=\left\{k_{1}, k_{2}, s\right\}^{\perp \perp}$. 
As $k_{1}, k_{2}, k_{3} \in p_{x}$, we find a line $s$ in $G_{x}$ such that $\left\{k_{i}, s\right\}_{x}^{\perp} \neq \emptyset$ if $s \neq k_{i}$, for each $i \in\{1,2,3\}$ and $\left\{k_{1}, k_{2}, s\right\}_{x}^{\perp}=\emptyset$. By Remark 3.8 and Lemma 3.6 the double perp $\left\{s, k_{i}\right\}^{\perp \perp}$ is minimal with respect to inclusion, if $s \neq k_{i}$, for $i \in\{1,2,3\}$.

Furthermore $\emptyset=\left\{k_{1}, k_{2}, s\right\}_{x}^{\perp} \subsetneq\left\{k_{1}, k_{2}\right\}_{x}^{\perp}$ implying that $\left\{k_{1}, k_{2}\right\}^{\perp \perp}=\mathbf{L}\left(\left\langle k_{1}, k_{2}\right\rangle\right)$ $=\left\{k_{1}, k_{2}\right\}_{x}^{\perp \perp} \subseteq\left\{k_{1}, k_{2}, s\right\}_{x}^{\perp \perp} \subseteq\left\{k_{1}, k_{2}, s\right\}^{\perp \perp}$. However, as $\left\{k_{1}, k_{2}, s\right\}_{x}^{\perp}$ is the empty graph, $\operatorname{dim}\left(\left\langle k_{1}, k_{2}, s\right\rangle^{\pi} \cap x^{\pi}\right)<2$ and thus $\left\langle k_{1}, k_{2}, s\right\rangle$ is a subspace of $x^{\pi}$ of dimension at least four. Hence $\left\langle k_{1}, k_{2}\right\rangle \varsubsetneqq\left\langle k_{1}, k_{2}, s\right\rangle$ and $s \nsubseteq\left\langle k_{1}, k_{2}\right\rangle$, so $\left\{k_{1}, k_{2}\right\}^{\perp \perp} \varsubsetneqq$ $\left\{k_{1}, k_{2}, s\right\}^{\perp \perp}$.

Proposition 4.6. Let $\Gamma \cong \mathbf{S}\left(V_{7}\right)$ and let $x \in \Gamma$. Any point $p_{x}$ of the interior space $\mathcal{G}_{x}$ on $x^{\perp}$ is contained in a unique point pof the interior space $\mathcal{G}$ of $\Gamma$. The resulting embedding of $\mathcal{G}_{x}$ into $\mathcal{G}$ turns $\mathcal{G}_{x}$ into a codimension two subspace of $\mathcal{G}$.

Proof. It remains to prove the claim about the codimension. This, however, follows from the fact that $\mathcal{G}_{x} \cong \mathbb{P}\left(V_{5}\right)$ and $\mathcal{G} \cong \mathbb{P}\left(V_{7}\right)$.

The analogue holds for arbitrary $n \geq 8$.

Proposition 4.7. Let $n \geq 8$, let $\Gamma \cong \mathbf{S}\left(V_{n}\right)$, and let $x \in \Gamma$. Any point $p_{x}$ of the interior space $\mathcal{G}_{x}$ on $x^{\perp}$ is contained in a unique point $p$ of the interior space $\mathcal{G}$ of $\Gamma$. The resulting embedding of $\mathcal{G}_{x}$ into $\mathcal{G}$ turns $\mathcal{G}_{x}$ into a codimension two subspace of $\mathcal{G}$.

Proof. Left to the reader as an exercise.

\section{The global space}

In this section we prove the main result, i.e., we prove that if $\Gamma$ is a connected graph which is locally isomorphic to $\mathbf{S}\left(V_{n}\right)$ for $n \geq 7$, then $\Gamma$ is isomorphic to $\mathbf{S}\left(V_{n+2}\right)$. For every vertex $\mathrm{x}$ of $\Gamma$ Proposition 3.12 allows us to construct the interior space $\mathcal{G}_{\mathrm{x}}$ on $\mathrm{x}^{\perp}$ isomorphic to $\mathbb{P}\left(V_{n}\right)$. Our approach towards a proof of the main theorem is via a construction of a global geometry on $\Gamma$ using the family $\left(\mathcal{G}_{\mathbf{x}}\right)_{\mathbf{x} \in \Gamma}$ of local interior spaces. This global geometry will turn out to be a projective space. Observe that so far geometric objects (points, lines, planes, etc.) only exist in the local interior spaces $\mathcal{G}_{\mathbf{x}}$ for $\mathbf{x} \in \Gamma$. For emphasis we call these objects local. Moreover, we index every local object by the vertex $\mathrm{x}$ whose interior space it belongs to. One task will be to show that there exists a well-defined notion of global objects in order to define our global geometry.

Notation. In this section we use the following convention for the fonts of variables. Each vertex $\mathbf{x}$ of a graph is denoted in boldface. If $\mathbf{x}$ and $\mathbf{y}$ are adjacent vertices in $\Gamma$, then the line of $\mathcal{G}_{\mathbf{x}}$ corresponding to the vertex $\mathbf{y}$ is denoted by $y_{\mathbf{x}}$. Moreover, we will use the notation $\mathbf{y}_{\mathbf{x}}$, if we want to emphasise that we consider the vertex $\mathbf{y}$ as an element of the induced subgraph $\mathbf{x}^{\perp}$ rather than $\Gamma$. So, for example, the symbol $\mathbf{y}_{\mathbf{x}}^{\perp}$ denotes the subgraph $\{\mathbf{x}, \mathbf{y}\}^{\perp}$. The interior space obtained from the graph $\mathbf{y}_{\mathbf{x}}^{\perp}$ will be denoted by $\mathcal{G}_{\mathbf{y}_{\mathbf{x}}}$. 
Definition 5.1. A global line of $\Gamma$ is a vertex of the graph $\Gamma$. The set of all global lines of $\Gamma$ is denoted by $\mathcal{L}_{\Gamma}$.

Let $\mathbf{x}, \mathbf{y}, \mathbf{z}, \mathbf{w} \in \Gamma$ such that $\mathbf{z} \perp \mathbf{x} \perp \mathbf{w} \perp \mathbf{y}$. Then $U_{z_{\mathbf{x}}, w_{\mathbf{x}}}^{\mathbf{x}}:=\left\langle z_{\mathbf{x}}, w_{\mathbf{x}}\right\rangle^{\pi}=z_{\mathbf{x}}^{\pi} \cap w_{\mathbf{x}}^{\pi}$ is a subspace of the interior space $\mathcal{G}_{\mathbf{x}}$ and of the interior space $\mathcal{G}_{\mathbf{x}_{\mathbf{z}}}$. Proposition 3.12 implies $\mathcal{G}_{\mathbf{x}_{\mathbf{z}}}=\mathcal{G}_{\mathbf{z}_{\mathbf{x}}}$, because both $\mathcal{G}_{\mathbf{x}_{\mathbf{z}}}$ and $\mathcal{G}_{\mathbf{z}_{\mathbf{x}}}$ are interior spaces of the same graph $\mathbf{x}^{\perp} \cap \mathbf{z}^{\perp}$. Hence by Propositions 4.6 and 4.7 this space $U_{z_{\mathbf{x}}, w_{\mathbf{x}}}^{\mathbf{x}}$ can be considered as a subspace of the interior space $\mathcal{G}_{\mathbf{z}}$, in fact of $x_{\mathbf{z}}^{\pi}$. For emphasis we denote $U_{z_{\mathbf{x}}, w_{\mathbf{x}}}^{\mathbf{x}}$ by $U_{z_{\mathbf{x}}, w_{\mathbf{x}}}^{\mathbf{z}}$ when considering it as a subspace of $\mathcal{G}_{\mathbf{z}}$. Altogether any local object contained in $\underset{z_{\mathbf{x}}, w_{\mathbf{x}}}{\mathbf{x}} \cap U_{x_{\mathbf{w}}, y_{\mathbf{w}}}^{\mathbf{x}}$ is a local object of each of the interior spaces $\mathcal{G}_{\mathbf{z}}, \mathcal{G}_{\mathbf{x}}, \mathcal{G}_{\mathbf{y}}, \mathcal{G}_{\mathbf{w}}$.

Proposition 5.2. Let $n \geq 7$ and let $\Gamma$ be a connected graph which is locally isomorphic to $\mathbf{S}\left(V_{n}\right)$. Then $\Gamma$ has diameter two. Moreover, if $\mathbf{z} \perp \mathbf{x} \perp \mathbf{y} \perp \mathbf{w}$ is a chain of vertices in $\Gamma$, then there exists $\mathbf{l} \in\{\mathbf{z}, \mathbf{y}, \mathbf{w}\}^{\perp}$.

Proof. Let $\mathbf{z} \perp \mathbf{x} \perp \mathbf{y} \perp \mathbf{w}$ be a chain of vertices in $\Gamma$. We will distinguish between the cases that $n \geq 8$ and $n=7$.

- $n \geq 8$ : The dimension of the intersection of $U_{z_{x}, y_{x}}^{\mathbf{y}}$ and $U_{x_{\mathbf{y}}, w_{\mathbf{y}}}^{\mathbf{y}}$ is at least two as $\operatorname{dim}\left(U_{z_{\mathbf{x}}, y_{\mathbf{x}}}^{\mathbf{y}}\right)=\operatorname{dim}\left(U_{x_{\mathbf{y}}, w_{\mathbf{y}}}^{\mathbf{y}}\right)=\operatorname{dim}\left(x_{\mathbf{y}}^{\pi}\right)+\operatorname{dim}\left(w_{\mathbf{y}}^{\pi}\right)-\operatorname{dim}\left(\left\langle x_{\mathbf{y}}^{\pi}, w_{\mathbf{y}}^{\pi}\right\rangle\right) \geq n-4$ and both $U_{z_{\mathbf{x}}, y_{\mathbf{x}}}^{\mathbf{y}}$ and $U_{x_{\mathbf{y}}, w_{\mathbf{y}}}^{\mathbf{y}}$ are subspaces of $x_{\mathbf{y}}^{\pi}$. Therefore we can choose an interior line $l_{\mathbf{y}}$ in $U_{z_{\mathbf{x}}, y_{\mathbf{x}}}^{\mathbf{y}} \cap U_{x_{\mathbf{y}}, w_{\mathbf{y}}}^{\mathbf{y}}$. This interior line $l_{\mathbf{y}}$ corresponds to a vertex $\mathbf{l}$ of $\Gamma$ and is adjacent to all of $\mathbf{x}, \mathbf{y}, \mathbf{z}, \mathbf{w}$.

- $n=7$ : In view of the preceding paragraph it remains to deal with the case where $U_{z_{\mathbf{x}}, y_{\mathbf{x}}}^{\mathbf{y}} \cap U_{x_{\mathbf{y}}, w_{\mathbf{y}}}^{\mathbf{y}}=p_{\mathbf{y}}$ is an interior point of $\mathcal{G}_{\mathbf{y}}$, whence also of each of the interior spaces $\mathcal{G}_{\mathbf{z}}, \mathcal{G}_{\mathbf{x}}, \mathcal{G}_{\mathbf{w}}$. Fix an interior line $n_{\mathrm{x}}$ of the at least three-dimensional subspace $U_{z_{\mathbf{x}}, y_{\mathbf{x}}}^{\mathbf{x}}$ incident to the interior point $p_{\mathbf{x}}$. Hence $n_{\mathbf{x}}$ corresponds to a vertex $\mathbf{n} \in \Gamma$ adjacent to $\mathbf{x}, \mathbf{y}$ and $\mathbf{z}$. Therefore we can consider the chain $\mathbf{z} \perp \mathbf{n} \perp \mathbf{y} \perp \mathbf{w}$ instead of $\mathbf{z} \perp \mathbf{x} \perp \mathbf{y} \perp \mathbf{w}$. Again there is an interior point $q_{\mathbf{n}}=U_{z_{\mathbf{n}}, y_{\mathbf{n}}}^{\mathbf{n}} \cap U_{n_{\mathbf{y}}, w_{\mathbf{y}}}^{\mathbf{n}}$ in $\mathcal{G}_{\mathbf{n}}$. The interior points $q_{\mathbf{y}}$ and $p_{\mathbf{y}}$ are different, because $p_{\mathbf{y}}$ lies on the two-dimensional subspace $n_{\mathbf{y}}$ and $q_{\mathbf{y}}$ is orthogonal to $n_{\mathbf{y}}$. Hence the span $l_{\mathbf{y}}=\left\langle p_{\mathbf{y}}, q_{\mathbf{y}}\right\rangle$ is a line contained in $w_{\mathbf{y}}^{\pi} \subseteq \mathcal{G}_{\mathbf{y}}$. This line $l_{\mathbf{y}}$ corresponds to a vertex $\mathbf{l}$ adjacent to $\mathbf{y}$ and $\mathbf{w}$.

We have constructed the chain $\mathbf{z} \perp \mathbf{n} \perp \mathbf{y} \perp \mathbf{l}$ in $\Gamma$ with the property that the dimension of the span $\left\langle n_{\mathbf{y}}, l_{\mathbf{y}}\right\rangle$ is three as the interior lines $n_{\mathbf{y}}$ and $l_{\mathbf{y}}$ intersect in the interior point $p_{\mathbf{y}}$ in $\mathcal{G}_{\mathbf{y}}$. Thus $\operatorname{dim}\left(U_{n_{\mathbf{y}}, l_{\mathbf{y}}}^{\mathbf{y}}\right)=n-3$ and therefore $\operatorname{dim}\left(U_{z_{\mathbf{n}}, y_{\mathbf{n}}}^{\mathbf{y}} \cap U_{n_{\mathbf{y}}, l_{\mathbf{y}}}^{\mathbf{y}}\right) \geq$ $n-4+n-3-n+2=n-5=2$. Hence there is a vertex $\mathbf{t} \in \Gamma$ adjacent to the vertices $\mathbf{z}, \mathbf{n}, \mathbf{y}, \mathbf{l}$. The vertices $\mathbf{y}$ and $\mathbf{z}$ correspond to interior lines $y_{\mathbf{t}}$ and $z_{\mathbf{t}}$ in $\mathcal{G}_{t}$ and the intersection of $y_{\mathbf{t}}^{\pi}$ and $z_{\mathrm{t}}^{\pi}$ is of dimension three. Furthermore the subspace $y_{\mathbf{t}}^{\pi} \cap z_{\mathbf{t}}^{\pi}$ contains the interior points $p_{\mathbf{t}}$ and $q_{\mathbf{t}}$ induced by $p_{\mathbf{y}}$, respectively $q_{\mathbf{y}}$. Hence the interior line $l_{\mathbf{t}}$ is incident to $U_{z_{\mathbf{t}}, y_{\mathbf{t}}}^{\mathbf{t}}$. Therefore $\mathbf{l}$ and $\mathbf{z}$ are adjacent in $\Gamma$, so that $\mathbf{z}$ and $\mathbf{w}$ have distance at most two.

In both cases it follows by induction that the connected graph $\Gamma$ has diameter 2 .

Our next goal is to define a notion of global points for $\Gamma$. The following observation is the reason why such a definition is possible. 
Let $\mathbf{x}, \mathbf{y}, \mathbf{z}$ be three mutually adjacent vertices of the graph $\Gamma$ and let $p_{\mathbf{x}}$ be an interior point of $\mathcal{G}_{\mathbf{x}}$ contained in the subspace $y_{\mathbf{x}}^{\pi} \cap z_{\mathbf{x}}^{\pi}$. Then $p_{\mathbf{y}_{\mathbf{x}}}=p_{\mathbf{x}} \cap \mathcal{L}_{\mathbf{y}}$ is an interior point of $\mathcal{G}_{\mathbf{y}_{\mathbf{x}}}$ and $p_{\mathbf{z}_{\mathbf{x}}}=p_{\mathbf{x}} \cap \mathcal{L}_{\mathbf{z}}$ is an interior point of $\mathcal{G}_{\mathbf{z}_{\mathbf{x}}}$ by Section 4 . Let $p_{\mathbf{y}}$ respectively $p_{\mathbf{z}}$ be the unique interior points of $\mathcal{G}_{\mathbf{y}}$, respectively $\mathcal{G}_{\mathbf{z}}$ containing $p_{\mathbf{y}_{\mathbf{x}}}$, respectively $p_{\mathbf{y}_{\mathbf{x}}}$.

As the intersection $y_{\mathrm{x}}^{\pi} \cap z_{\mathrm{x}}^{\pi}$ is at least three-dimensional in $\mathcal{G}_{\mathbf{x}} \cong \mathbb{P}\left(V_{n}\right)$ and the point $p_{\mathbf{x}}$ is contained in this intersection, the subspace $U_{y_{\mathbf{x}}, z_{\mathbf{x}}}^{x}$ contains distinct interior lines $g_{\mathbf{x}}^{1}$ and $g_{\mathbf{x}}^{2}$ incident to the interior point $p_{\mathbf{x}}$. Hence the vertices $\mathbf{g}^{1}$ and $\mathbf{g}^{2}$ are elements of the interior points $p_{\mathbf{y}_{\mathbf{x}}}$ and $p_{\mathbf{z}_{\mathbf{x}}}$. Thus $\mathbf{g}^{1}, \mathbf{g}^{2} \in p_{\mathbf{y}}$ and $\mathbf{g}^{1}, \mathbf{g}^{2} \in p_{\mathbf{z}}$. Consequently, $\mathbf{g}^{1}, \mathbf{g}^{2} \in p_{\mathbf{y}} \cap \mathcal{L}_{\mathbf{z}}=p_{\mathbf{y}_{\mathbf{z}}}$ and $\mathbf{g}^{1}, \mathbf{g}^{2} \in p_{\mathbf{z}} \cap \mathcal{L}_{\mathbf{y}}=p_{\mathbf{z}_{\mathbf{y}}}$. Hence $p_{\mathbf{y}_{\mathbf{z}}}=p_{\mathbf{z}_{\mathbf{y}}}$, as both $p_{\mathbf{y}_{\mathbf{z}}}$ and $p_{\mathbf{z}_{\mathbf{y}}}$ are interior points of the interior space $\mathcal{G}_{\mathbf{y}_{\mathbf{z}}}=\mathcal{G}_{\mathbf{z}_{\mathbf{y}}}$ of the induced subgraph $\mathbf{y}^{\perp} \cap \mathbf{z}^{\perp}$ by Proposition 4.3 which have more than one element in common. Thus we have proved the following statement.

Lemma 5.3. Let $\mathbf{x}, \mathbf{y}, \mathbf{z}$ be mutually adjacent vertices of $\Gamma$ and let $p_{\mathbf{x}}$ be an interior point of $\mathcal{G}_{\mathbf{x}}$ perpendicular to $y_{\mathbf{x}}$ and $z_{\mathbf{x}}$. Then the interior points $p_{\mathbf{y}}$ and $p_{\mathbf{z}}$ correspond to each other, i.e., $p_{\mathbf{y}_{\mathbf{z}}}=p_{\mathbf{z}_{\mathbf{y}}}$.

Definition 5.4. A global point $p$ of $\Gamma$ is a set of vertices of the graph $\Gamma$ satisfying

$$
p=p_{\mathbf{x}} \cup \bigcup_{\mathbf{l} \in \mathbf{x}^{\perp}}\left\{p_{\mathbf{l}} \in \mathcal{P}_{\mathbf{l}} \mid p_{\mathbf{x}_{\mathbf{1}}} \subseteq p_{\mathbf{l}}\right\}
$$

for some vertex $\mathbf{x}$ of $\Gamma$ and some interior point $p_{\mathbf{x}}$ of the interior space $\mathcal{G}_{\mathbf{x}}$. The set of all global points of $\Gamma$ is denoted by $\mathcal{P}_{\Gamma}$.

A global point is stable under iteration of the above process. Indeed, let $\mathbf{x} \in \Gamma$ and $p_{\mathbf{x}} \in \mathcal{P}_{\mathbf{x}}$ and define

$$
\begin{gathered}
p_{0}:=p_{\mathbf{x}} \cup \bigcup_{\mathbf{l} \in \mathbf{x}^{\perp}}\left\{p_{\mathbf{l}} \in \mathcal{P}_{\mathbf{l}} \mid p_{\mathbf{x}_{\mathbf{l}}} \subseteq p_{\mathbf{l}}\right\} \quad \text { and } \\
p_{1}:=p_{\mathbf{x}} \cup \bigcup_{\mathbf{l} \in \mathbf{x}^{\perp}}\left\{p_{\mathbf{l}} \in \mathcal{P}_{\mathbf{l}} \mid p_{\mathbf{x}_{\mathbf{1}}} \subseteq p_{\mathbf{l}}\right\} \cup \bigcup_{\mathbf{k} \perp \mathbf{l} \perp \mathbf{x}}\left\{p_{\mathbf{k}} \in \mathcal{P}_{\mathbf{k}} \mid p_{\mathbf{x}_{\mathbf{l}}} \subseteq p_{\mathbf{l}}, p_{\mathbf{l}_{\mathbf{k}}} \subseteq p_{\mathbf{k}}\right\} .
\end{gathered}
$$

Certainly $p_{0} \subseteq p_{1}$. If $p_{1} \neq p_{0}$, then there exists a vertex $\mathbf{w} \in p_{1}$ such that $\mathbf{w} \notin p_{0}$. By construction there exist a chain of vertices $\mathbf{x} \perp \mathbf{l} \perp \mathbf{k} \perp \mathbf{w}$ in $\Gamma$ and interior points $p_{\mathbf{l}}$ and $p_{\mathbf{k}}$ with $p_{\mathbf{x}_{1}} \subseteq p_{\mathbf{l}}, p_{\mathbf{l}_{\mathbf{k}}} \subseteq p_{\mathbf{k}}$ and $\mathbf{w} \in p_{\mathbf{k}}$. Due to the proof of Proposition 5.2 there are a vertex $\mathbf{z} \in\{\mathbf{x}, \mathbf{l}, \mathbf{w}\}^{\perp}$ and a path $\mathbf{k} \perp \mathbf{c}^{1} \perp \cdots \perp \mathbf{c}^{n} \perp \mathbf{z}$ in $\{\mathbf{l}, \mathbf{w}\}^{\perp}$. Since the interior line $c_{\mathbf{k}}^{1}$ is perpendicular to $w_{\mathbf{k}}$, there is a interior point $p_{\mathbf{c}^{1}} \supseteq p_{\mathbf{k}_{\mathbf{c}^{1}}}$ incident to $w_{\mathbf{c}^{1}}$. By Lemma 5.3 we have $p_{\mathbf{l}_{\mathbf{c}^{1}}} \subseteq p_{\mathbf{c}^{1}}$. Arguing along the path $\mathbf{k} \perp \mathbf{c}^{1} \perp \cdots \perp \mathbf{c}^{n} \perp \mathbf{z}$, we obtain $p_{\mathbf{l}_{\mathbf{z}}} \subseteq p_{\mathbf{z}}, w_{\mathbf{z}} \in p_{\mathbf{z}}$. Therefore $p_{\mathbf{x}_{\mathbf{z}}} \subseteq p_{\mathbf{z}}$, so $\mathbf{w} \in p_{0}$, a contradiction.

This consideration has two immediate consequences.

Proposition 5.5. Let $p$ be a global point and $\mathrm{x}$ be vertex of $\Gamma$.

- The intersection $\mathcal{L}_{\mathbf{x}} \cap p$ is either empty or an interior point of $\mathcal{G}_{\mathbf{x}}$.

- The global point $p$ does not depend on the starting interior point $p_{\mathbf{x}} \subseteq p$. 
The triple $\mathcal{G}_{\Gamma}=\left(\mathcal{P}_{\Gamma}, \mathcal{L}_{\Gamma}, \supset\right)$ is a point-line geometry, called the global geometry on $\Gamma$.

Proposition 5.6. The point-line geometry $\mathcal{G}_{\Gamma}=\left(\mathcal{P}_{\Gamma}, \mathcal{L}_{\Gamma}, \supset\right)$ is a projective space.

Proof. We have to show that the geometry $\mathcal{G}_{\Gamma}$ satisfies the axioms of a projective space, i.e., $\mathcal{G}_{\Gamma}$ is linear and satisfies the axiom of Veblen and Young. Note that $\mathcal{G}_{\Gamma}$ is automatically thick, because the spaces $\mathcal{G}_{\mathbf{x}}, \mathbf{x} \in \Gamma$, are.

We start with proving linearity. Let $p$ and $q$ be two distinct global points of $\mathcal{G}_{\Gamma}$ and fix two global lines $\mathbf{l} \in p$ and $\mathbf{m} \in q$. By Proposition 5.2 there exists a vertex $\mathbf{z} \in \Gamma$ adjacent to $\mathbf{l}$ and $\mathbf{m}$. Hence Proposition 5.5 implies that $p \cap \mathcal{L}_{\mathbf{z}}=p_{\mathbf{z}}$ and $q \cap \mathcal{L}_{\mathbf{z}}=q_{\mathbf{z}}$ are interior points of $\mathcal{G}_{\mathbf{z}}$. By Proposition 3.12, i.e., since the projective space $\mathcal{G}_{\mathbf{z}}$ is linear, there is a unique interior line $k_{\mathbf{z}}$ of $\mathcal{G}_{\mathbf{z}}$ incident to both $p_{\mathbf{z}}$ and $q_{\mathbf{z}}$. Thus we have found a global line $\mathbf{k}$ joining the global points $p$ and $q$. If $\mathbf{l}$ were another global line joining the global points $p$ and $q$, Proposition 5.2 would give us a vertex $\mathbf{m} \in\{\mathbf{k}, \mathbf{l}\}$ and a contradiction to the linearity of $\mathcal{G}_{\mathbf{m}}$. So $\mathcal{G}_{\Gamma}$ is linear.

We now turn to the axiom of Veblen and Young. Let $a, b, c, d, e$ be global points of $\mathcal{G}_{\Gamma}$ such that the global points $a, b, c$ and $a, d, e$ are collinear triples on distinct global lines, say $\mathbf{l}^{a, b, c}$ and $\mathbf{k}^{a, d, e}$. We need to prove that the line $\mathbf{m}^{b, d}$ joining $b$ and $d$ intersects the line $\mathbf{n}^{c, e}$ joining $c$ and $e$. By Proposition 5.2 there exists $\mathbf{z} \in \Gamma$ satisfying $\mathbf{m}^{b, d} \perp \mathbf{z} \perp$ $\mathbf{n}^{c, e}$. The claim follows from local analysis of the interior space $\mathcal{G}_{\mathbf{z}}$ and Propositions 3.12 and 5.5. Indeed the interior space $\mathcal{G}_{\mathbf{z}}$ contains the interior lines $m_{\mathbf{z}}^{b, d}$ and $n_{\mathbf{z}}^{c, e}$ and the interior points $b_{\mathbf{z}}, c_{\mathbf{z}}, d_{\mathbf{z}}, e_{\mathbf{z}}$. Moreover, the space $\mathcal{G}_{\mathbf{z}}$ contains the lines joining the pair $b_{\mathbf{z}}, c_{\mathbf{z}}$ and the pair $d_{\mathbf{z}}, e_{\mathbf{z}}$. This means that $l_{\mathbf{z}}^{a, b, c}$ and $k_{\mathbf{z}}^{a, d, e}$ are lines of $\mathcal{G}_{\mathbf{z}}$. Therefore the interior point $a_{\mathbf{z}}=a \cap \mathcal{L}_{\mathbf{z}}$ exists in $\mathcal{G}_{\mathbf{z}}$. By the validity of the axiom of Veblen and Young in the projective space $\mathcal{G}_{\mathbf{z}}$ the interior lines $m_{\mathbf{z}}^{b, d}$ and $n_{\mathbf{z}}^{c, e}$ intersect in a point $f_{\mathbf{z}}$. Extending this local point to a global point $f$ finishes the proof.

Definition 5.7. Let $\mathrm{x} \in \Gamma$. Then $\left\langle\mathrm{x}^{\perp}\right\rangle$ denotes the pair $\left(\mathcal{P}^{\mathrm{x}}, \mathcal{L}_{\mathbf{x}}\right) \subseteq\left(\mathcal{P}_{\Gamma}, \mathcal{L}_{\Gamma}\right)$ where $\mathcal{P}^{\mathbf{x}}$ contains the global points which have a non-empty intersection with $\mathcal{L}_{\mathbf{x}}$, i.e. $p \in \mathcal{P}^{\mathbf{x}}$ if and only if $p \cap \mathcal{L}_{\mathbf{x}} \neq \emptyset$. For a global point $p$ we denote by $\left\langle p^{\perp}\right\rangle$ the pair $\left(\mathcal{P}^{p}, \mathcal{L}^{p}\right):=$ $\left(\bigcup_{\mathbf{x} \in p} \mathcal{P}^{\mathbf{x}}, \bigcup_{\mathbf{x} \in p} \mathcal{L}_{\mathbf{x}}\right) \subseteq\left(\mathcal{P}_{\Gamma}, \mathcal{L}_{\Gamma}\right)$.

It turns out that for each $\left\langle\mathbf{x}^{\perp}\right\rangle$ is a hyperline and each $\left\langle p^{\perp}\right\rangle$ is a hyperplane of $\mathcal{G}_{\Gamma}$, cf. Propositions 5.9 and 5.11.

Lemma 5.8. Let $n \geq 7$. The geometries $\left\langle\mathbf{x}^{\perp}\right\rangle$ and $\left\langle p^{\perp}\right\rangle$ are subspaces of $\mathcal{G}_{\Gamma}$. Moreover, $\left\langle\mathbf{x}^{\perp}\right\rangle \cong \mathcal{G}_{\mathbf{x}}$.

Proof. We start with proving that $\left\langle\mathbf{x}^{\perp}\right\rangle$ is a subspace. We have to show that for distinct $p, q \in \mathcal{P}^{\mathbf{x}}$ the line $\mathbf{l} \in \mathcal{L}_{\Gamma}$ joining $p$ and $q$ lies in $\mathcal{L}_{\mathbf{x}}$. Proposition 5.5 yields interior points $p_{\mathbf{x}}=p \cap \mathcal{L}_{\mathbf{x}}$ and $q_{\mathbf{x}}=q \cap \mathcal{L}_{\mathbf{x}}$ of the interior space $\mathcal{G}_{\mathbf{x}}$. By Proposition 3.12 there exists a unique interior line $l_{\mathbf{x}}$ incident to both $p_{\mathbf{x}}$ and $q_{\mathbf{x}}$. Hence $\mathbf{l} \in \mathcal{L}_{\mathbf{x}}$. The map $\left\langle\mathbf{x}^{\perp}\right\rangle \rightarrow \mathcal{G}_{\mathbf{x}}: p \mapsto p \cap \mathcal{L}_{\mathbf{x}}$ and $\mathbf{l} \mapsto \mathbf{l}$ for every $p \in \mathcal{P}^{\mathbf{x}}$ and $\mathbf{l} \in \mathcal{L}_{\mathbf{x}}$ is the desired isomorphism. 
Now we turn to $\left\langle p^{\perp}\right\rangle$. We have to show that for distinct global points $a, b \in \mathcal{P}^{p}$, the global line $\mathbf{l}$ joining $a$ and $b$ is an element of the line set $\mathcal{L}^{p}$. Let $\mathbf{x}, \mathbf{y} \in p$ such that $a \in \mathcal{P}^{\mathbf{x}}$ and $b \in \mathcal{P}^{\mathbf{y}}$. Fix two interior lines $h_{\mathbf{x}}^{a} \in a_{\mathbf{x}}$ and $h_{\mathbf{y}}^{b} \in b_{\mathbf{y}}$. By Lemma 5.2 there exists a vertex $\mathbf{z} \in \Gamma$ adjacent to $\mathbf{x}$ and $\mathbf{y}$. This yields the chain of vertices $\mathbf{h}^{a} \perp \mathbf{x} \perp \mathbf{z} \perp \mathbf{y} \perp \mathbf{h}^{b}$ in the graph $\Gamma$. The subspace $U_{x_{\mathbf{z}}, y_{\mathbf{z}}}^{\mathbf{x}} \cap\left(h_{\mathbf{x}}^{a}\right)^{\pi}$ has at least dimension three and therefore this intersection contains an interior line $g_{\mathbf{x}}$. Thus we obtain the chain of vertices $\mathbf{g} \perp$ $\mathbf{h}^{a} \perp \mathbf{x} \perp \mathbf{g} \perp \mathbf{y} \perp \mathbf{h}^{b}$ in $\Gamma$. Therefore, by passing from $\mathbf{h}^{a} \perp \mathbf{x} \perp \mathbf{z} \perp \mathbf{y} \perp \mathbf{h}^{b}$ to g $\perp \mathbf{h}^{a} \perp \mathbf{x} \perp \mathbf{g} \perp \mathbf{y} \perp \mathbf{h}^{b}$ if necessary, we can assume that $a \cap \mathcal{L}_{\mathbf{z}}$ is an interior point of $\mathcal{G}_{\mathbf{z}}$ and that $\mathbf{h}^{a}$ is adjacent to $\mathbf{z}$ in $\Gamma$. The intersection $a_{\mathbf{z}}^{\pi} \cap U_{x_{\mathbf{z}}, y_{\mathbf{z}}}^{\mathbf{z}}=: H_{x_{\mathbf{z}}, y_{\mathbf{z}}, a_{\mathbf{z}}}^{\mathbf{z}}$ has dimension at least $n-4$ and is a subspace of the hyperline $y_{\mathbf{z}}^{\pi}$. Hence $H_{x_{\mathbf{z}}, y_{\mathbf{z}}, a_{\mathbf{z}}}^{\mathbf{y}} \cap b_{\mathbf{y}}^{\pi}$ has dimension at least $n-5$, and thus contains a interior line $t_{\mathbf{y}}$. Considering the chain of vertices $\mathbf{t} \perp \mathbf{h}^{a} \perp \mathbf{x} \perp \mathbf{t} \perp \mathbf{y} \perp \mathbf{h}^{b} \perp \mathbf{t}$ instead of $\mathbf{z} \perp \mathbf{h}^{a} \perp \mathbf{x} \perp \mathbf{z} \perp \mathbf{y} \perp \mathbf{h}^{b}$, if necessary, we can additionally assume that $b \cap \mathcal{L}_{\mathbf{z}}$ is non-empty and that $\mathbf{h}^{b} \perp \mathbf{z}$. Form the path $\mathbf{z} \perp \mathbf{h}^{a} \perp \mathbf{x} \perp \mathbf{z} \perp \mathbf{y} \perp \mathbf{h}^{b} \perp \mathbf{z}$ in $\Gamma$ we obtain that both interior lines $h_{\mathbf{z}}^{a}$ and $h_{\mathbf{z}}^{b}$ are contained in the polar of $p_{\mathbf{z}}$. Thus the interior line $l_{\mathbf{z}}^{a, b}$ joining $a_{\mathbf{z}}$ and $b_{\mathbf{z}}$ is contained in $p_{\mathbf{z}}^{\pi}$, too. Consequently, there is a vertex $\mathbf{w} \in p$ such that $l_{\mathbf{z}}^{a, b} \subseteq w_{\mathbf{z}}^{\pi}$ implying $\mathbf{l}^{a, b} \in \mathcal{L}_{\mathbf{w}} \subseteq \mathcal{L}^{p}$. Hence $\mathbf{l}^{a, b} \in\left\langle p^{\perp}\right\rangle$.

Proposition 5.9. Let $n \geq 7$ and let $\mathbf{x} \in \Gamma$. Then $\left\langle\mathbf{x}^{\perp}\right\rangle$ is a hyperline (i.e., a codimension two subspace) of $\mathcal{G}_{\Gamma}$.

Proof. The subspace $\left\langle\mathbf{x}^{\perp}\right\rangle$ has codimension at least two, because none of the global lines $\mathbf{h} \in \mathcal{L}_{\mathbf{x}}$ intersects the global line $\mathbf{x}$. It remains to show that an arbitrary plane $E$ of $\mathcal{G}_{\Gamma}$ intersects the subspace $\left\langle\mathbf{x}^{\perp}\right\rangle$ nontrivially. Let $\mathbf{k}$ and $\mathbf{l}$ be two distinct lines in $E$. Then $E=\langle\mathbf{k}, \mathbf{l}\rangle$ and the two global lines $\mathbf{k}$ and $\mathbf{l}$ intersect in a global point $d$, thus $\mathbf{k}, \mathbf{l} \in d$. By Proposition 5.2 there exists a vertex $\mathbf{z} \in \Gamma$ adjacent to $\mathbf{k}$ and $\mathbf{l}$. Moreover the intersection $k_{\mathbf{z}}^{\pi} \cap\left\langle k_{\mathbf{z}}, l_{\mathbf{z}}\right\rangle$ contains an interior point $p_{\mathbf{z}}$. Let $\mathbf{l}^{p, d}$ be the global line of $\mathcal{G}_{\Gamma}$ joining the two global points $p$ and $d$ and $\mathbf{h}$ be a global line incident to the point $p$ and adjacent to the vertices $\mathbf{k}$ and $\mathbf{z}$. By Proposition 5.2 there again exists a vertex $\mathbf{y}$ such that $\mathbf{h} \perp \mathbf{y} \perp \mathbf{x}$. Proposition 5.2 also yields a vertex $\mathbf{s} \in\{\mathbf{k}, \mathbf{h}, \mathbf{x}\}^{\perp}$. As the interior space $\mathcal{G}_{\mathbf{s}}$ contains the two local points $p_{\mathbf{s}}=p \cap \mathcal{L}_{\mathbf{s}}$ and $d_{\mathbf{s}}=d \cap \mathcal{L}_{\mathbf{s}}$ the vertex $\mathbf{l}^{p, d}$ is adjacent to $\mathbf{s}$, too. Furthermore $\operatorname{dim}\left(x_{\mathbf{s}}^{\pi} \cap\left\langle k_{\mathbf{s}}, l_{\mathbf{s}}^{p, d}\right\rangle\right) \geq n-2+3-n=1$ implying that the intersection $\left.x_{\mathbf{s}}^{\pi} \cap\left\langle k_{\mathbf{s}}, l_{\mathbf{s}}^{p, d}\right\rangle\right)$ contains an interior point $q_{\mathbf{s}}$ of $\mathcal{G}_{\mathbf{s}}$. Consequently, the global plane $E=\langle\mathbf{k}, \mathbf{l}\rangle=\left\langle\mathbf{k}, \mathbf{l}^{p, d}\right\rangle$ intersects the subspace $\left\langle x^{\perp}\right\rangle$ in the global point $q$.

Corollary 5.10. The projective space $\mathcal{G}_{\Gamma}$ has vector space dimension $n+2$.

Proof. Indeed, the codimension two subspace $\left\langle\mathbf{x}^{\perp}\right\rangle \cong \mathcal{G}_{\mathbf{x}}$ has vector space dimension $n$.

Proposition 5.11. Let $n \geq 7$ and let $p$ be a global point of $\Gamma$. Then $\left\langle p^{\perp}\right\rangle$ is a hyperplane of $\mathcal{G}_{\Gamma}$.

Proof. Certainly the subspace $\left\langle p^{\perp}\right\rangle$ is a proper subspace of $\mathcal{G}_{\Gamma}$. Indeed, $p \notin\left\langle p^{\perp}\right\rangle$ as $p \cap \mathcal{L}_{\mathbf{x}}=\emptyset$ for each vertex $\mathbf{x} \in p$. It remains to prove that each global line meets $\left\langle p^{\perp}\right\rangle$. Let $\mathbf{l} \in \Gamma$ and let $\mathbf{k}$ be a global line of $p$. By Lemma 5.2 there exists a line $\mathbf{z} \in \Gamma$ satisfying 
$\mathbf{k} \perp \mathbf{z} \perp \mathbf{l}$. The orthogonal space $p_{\mathbf{z}}^{\pi}$ of $p_{\mathbf{z}}$ is a hyperplane of $\mathcal{G}_{\mathbf{z}}$. Therefore the interior line $l_{\mathbf{z}}$ intersects $p_{\mathbf{z}}^{\pi}$ in at least one interior point $q_{\mathbf{z}}$. Hence $\mathbf{l}$ is incident to the global point $q$. Moreover, there exists $\mathbf{m} \in p$ such that $m_{\mathbf{z}}^{\pi}$ contains the interior point $q_{\mathbf{z}}$. This implies $q \cap \mathcal{L}_{\mathbf{m}} \neq \emptyset$, whence $q$ is a point of the subspace $\left\langle p^{\perp}\right\rangle$ incident to 1 .

Proposition 5.12. Let $n \geq 7$ and let $\Gamma$ be a connected graph locally $\mathbf{S}\left(V_{n}\right)$. The pointline geometry $\mathcal{G}_{\Gamma}=\left(\mathcal{P}_{\Gamma}, \mathcal{L}_{\Gamma}\right)$ is isomorphic to $\mathbb{P}\left(V_{n+2}\right)$.

Proof. In view of Corollary 5.10 the only remaining question is the one for the isomorphism type of the underlying field. That field, however, is equal to the field underlying the projective spaces $\mathcal{G}_{\mathbf{x}}, \mathbf{x} \in \Gamma$.

Lemma 5.13. Let $H$ be a hyperline of $\mathcal{G}_{\Gamma}$. Then there exists a unique vertex $\mathbf{h} \in \Gamma$ satisfying $H=\left\langle\mathbf{h}^{\perp}\right\rangle$.

Proof. Let $\mathbf{k}$ be a global line in the hyperline $H$. By Lemma 5.9 the subspace $\left\langle\mathbf{k}^{\perp}\right\rangle$ is a hyperline of $\mathcal{G}_{\Gamma}$. Moreover, the intersection $L:=\left\langle\mathbf{k}^{\perp}\right\rangle \cap H$ is a hyperline of $H$, because $\mathbf{k}$ and $\left\langle\mathbf{k}^{\perp}\right\rangle$ intersect trivially. Therefore $L$ is also a hyperline of $\left\langle\mathbf{k}^{\perp}\right\rangle$ and induces a local hyperline $L_{\mathbf{k}} \cong L$ in the interior space $\mathcal{G}_{\mathbf{k}} \cong\left\langle\mathbf{k}^{\perp}\right\rangle$. Thus $L_{\mathbf{k}}=l_{\mathbf{k}}^{\pi}$ for some interior line $l_{\mathbf{k}}$. Obviously, $\mathbf{k} \in\left\langle\mathbf{l}^{\perp}\right\rangle$ and $L \subseteq\left\langle\mathbf{l}^{\perp}\right\rangle$. By dimension arguments we obtain $H=\langle\mathbf{k}, L\rangle=\left\langle\mathbf{l}^{\perp}\right\rangle$.

Let $\mathbf{m} \in \Gamma$ another vertex satisfying $H=\left\langle\mathbf{m}^{\perp}\right\rangle$. By Proposition 5.2 there exists $\mathbf{z} \in$ $\Gamma$ satisfying $\mathbf{l} \perp \mathbf{z} \perp \mathbf{m}$. The equality $\left\langle\mathbf{l}^{\perp}\right\rangle=\left\langle\mathbf{m}^{\perp}\right\rangle$ implies $\left\langle\mathbf{l}^{\perp}\right\rangle \cap\left\langle\mathbf{z}^{\perp}\right\rangle=\left\langle\mathbf{m}^{\perp}\right\rangle \cap\left\langle\mathbf{z}^{\perp}\right\rangle$. Local analysis of $\mathcal{G}_{\mathbf{z}} \cong\left\langle\mathbf{z}^{\perp}\right\rangle$ (cf. Lemma 5.8) yields $\mathbf{l}=\mathbf{m}$.

Lemma 5.14. Let $P$ be a hyperplane of $\mathcal{G}_{\Gamma}$. Then there exist a unique global point $p$ of $\mathcal{G}_{\Gamma}$ satisfying $P=\left\langle p^{\perp}\right\rangle$.

Proof. Let $\mathbf{k}$ be a global line of the hyperplane $P$. By Lemma 5.9 the subspace $\left\langle\mathbf{k}^{\perp}\right\rangle$ is hyperline of $\mathcal{G}_{\Gamma}$. The intersection $L:=\left\langle\mathbf{k}^{\perp}\right\rangle \cap P$ is a hyperplane of $\left\langle\mathbf{k}^{\perp}\right\rangle \cong \mathcal{G}_{\mathbf{k}}$. Thus $L$ induces a unique local hyperplane $L_{\mathbf{k}}$ in $\mathcal{G}_{\mathbf{k}}$. Hence $L_{\mathbf{k}}$ is the orthogonal space of some interior point $p_{\mathbf{k}}$. Consequently, $\mathbf{k} \in\left\langle p^{\perp}\right\rangle$ and $L \subseteq\left\langle p^{\perp}\right\rangle$. Hence by dimension arguments $P=\langle\mathbf{k}, L\rangle=\left\langle p^{\perp}\right\rangle$. Uniqueness of $p$ follows by an argument as given in the proof of Lemma 5.13.

Denote the set of all hyperplanes of the projective geometry $\mathcal{G}_{\Gamma}$ with $\mathcal{H}_{\Gamma}$. Define

$$
\begin{aligned}
\pi_{\Gamma}: \mathcal{P}_{\Gamma} \cup \mathcal{H}_{\Gamma} & \rightarrow \mathcal{P}_{\Gamma} \cup \mathcal{H}_{\Gamma} \\
p & \mapsto\left\langle p^{\perp}\right\rangle \\
\left\langle p^{\perp}\right\rangle & \mapsto p .
\end{aligned}
$$

Let $\mathbf{S}\left(\mathcal{G}_{\Gamma}\right)$ be the line graph of $\mathcal{G}_{\Gamma}=\left(\mathcal{P}_{\Gamma}, \mathcal{L}_{\Gamma}\right)$. This means the vertex set of $\mathbf{S}\left(\mathcal{G}_{\Gamma}\right)$ is the set of global lines of $\mathcal{G}_{\Gamma}$ in which two distinct global lines $\mathbf{k}$ and $\mathbf{l}$ are adjacent if and only if $\mathbf{k} \in\left\langle\mathbf{l}^{\perp}\right\rangle=\pi_{\Gamma}(\mathbf{l})$ or, equivalently, if and only if $\mathbf{l} \in\left\langle\mathbf{k}^{\perp}\right\rangle=\pi_{\Gamma}(\mathbf{k})$.

Proposition 5.15. The graph $\Gamma$ is isomorphic to the line graph $\mathbf{S}\left(\mathcal{G}_{\Gamma}\right)$ of $\mathcal{G}_{\Gamma}$. 
Proof. The identity map $\Gamma \rightarrow \mathbf{S}\left(\mathcal{G}_{\Gamma}\right)$ is an isomorphism.

The Main Theorem is proved.

Acknowledgements. The authors express their gratitude to Antonio Pasini and to an anonymous referee for several very helpful comments and suggestions.

\section{References}

[1] K. Altmann, Centralisers of fundamental subgroups. PhD thesis, TU Darmstadt 2007, http://elib.tu-darmstadt.de/diss/000875/masterbook.pdf.

[2] K. Altmann, R. Gramlich, On the hyperbolic unitary geometry. J. Algebr. Comb., to appear.

[3] F. Buekenhout, X. Hubaut, Locally polar spaces and related rank 3 groups. J. Algebra 45 (1977), 391-434. MR0460155 (57 \#151) Zbl 0351.05021

[4] A. M. Cohen, H. Cuypers, R. Gramlich, Local recognition of non-incident point-hyperplane graphs. Combinatorica 25 (2005), 271-296. MR2141659 (2005m:05190) Zbl 1100.05080

[5] T. De Medts, R. Gramlich, M. Horn, Iwasawa decompositions of split Kac-Moody groups. J. Lie Theory 19 (2009), 311-337.

[6] R. Gramlich, Line-hyperline pairs of projective spaces and fundamental subgroups of linear groups. Adv. Geom. 4 (2004), 83-103. MR2155367 (2006e:51010) Zbl 1046.51001

[7] R. Gramlich, Defining amalgams of compact Lie groups. J. Lie Theory 16 (2006), 1-18. MR2196409 (2007g:22001) Zbl 1103.22003

[8] R. Gramlich, Developments in finite Phan theory. Incidence Geom., to appear. Eprint arXiv:0710.0034

Received 14 October, 2007

K. Altmann, c/o Holger Grothe, Fachbereich Mathematik, TU Darmstadt, Schloßgartenstraße 7, 64289 Darmstadt, Germany

Email: altmann@mathematik.tu-darmstadt.de

R. Gramlich, Fachbereich Mathematik, TU Darmstadt, Schloßgartenstraße 7, 64289 Darmstadt, Germany

Email: gramlich@mathematik.tu-darmstadt.de

Second author's alternative address: R. Gramlich, School of Mathematics, University of Birmingham, Edgbaston, Birmingham B15 2TT, United Kingdom

Email: ralfg@maths.bham.ac.uk 